\title{
INTRACRANIAL HYPOTENSION IN A SLE PATIENT
}

\author{
Mariana Oliveira Miranda ${ }^{1, \star}$, Gustavo Luis Behrens Pinto ${ }^{1}$, Renata Borges de Lima ${ }^{1}$, Maria de Lourdes Castro de Oliveira \\ Figueiroa ${ }^{1}$, Alisson Regis de Santana ${ }^{1}$, Viviane Leal Novais ${ }^{1}$, Victor Pereira Mattos ${ }^{1}$, Tamires Cristina Martins de Vasconcelos ${ }^{1}$, \\ Mittermayer Barreto Santiago ${ }^{1}$
}

1.Universidade Federal da Bahia, Salvador (BA), Brazil.

*Corresponding author: marianam_o@hotmail.com

\section{BACKGROUND}

Spontaneous intracranial hypotension ( $\mathrm{SIH}$ ) was described for the first time in 1938. Proposed mechanisms for its development are occult dural laceration, increased absorption and low cerebrospinal fluid (CSF) production. Its incidence is estimated at $5 / 100,000$ inhabitants/year, with a predominance of females. The main symptom is orthostatic headache, which worsens in an upright position, with the stimulus of coughing and the Valsalva maneuver's performance. Also remarkable is its resistance to the use of analgesics. The authors describe a rare case of $\mathrm{SIH}$ in a patient with systemic lupus erythematosus (SLE).

\section{CASE REPORT}

A 35-year-old female, SLE patient, was admitted to our hospital reporting a headache lasting 20 days. It was associated with phonophobia, worsening in orthostasis, and refractory to analgesics. She also reported worsening polyarthralgia. Physical examination was normal. Laboratory investigation showed anemia and elevated C-reactive protein. Cerebrospinal fluid (CSF) study showed withdrawal pressure below $10 \mathrm{~cm} \mathrm{H} 2 \mathrm{O}$ with protein content of the CSF $74 \mathrm{mg} / \mathrm{dL}$ (rr: $15-45 \mathrm{mg} / \mathrm{dL}$ ). Brain magnetic resonance imaging (MRI) showed left frontotemporoparietal subdural hematoma and diffuse and pachymeningeal thickening. Computed tomography myelography identified numerous foraminal dural diverticula at dorsal and cervical levels corroborating the diagnosis of SLE-associated SIH. Therapy with corticosteroid $1 \mathrm{mg} / \mathrm{kg} /$ day was instituted with improvement of her symptoms.

\section{CONCLUSION}

The diagnosis of $\mathrm{SIH}$ requires at least one of the following signs: low CSF pressure (opening pressure below $50 \mathrm{mmHg}$ or $10 \mathrm{~cm} \mathrm{H2O}$ ), evidence of CSF leakage (CT myelography, conventional myelography, or radionuclide cisternography), or diffuse pachymeningeal enhancement on brain MRI. Clinically, in addition to orthostatic headache, there may be hearing impairment, tinnitus, dizziness, fever, anorexia, nausea, vomiting, photophobia, diplopia, visual field alteration, cranial nerve palsies, back and cervical pain. There are only five cases of SIH in SLE patients described in the literature and it has been suggested that the fragility of the dura mater due to the deposit of immunocomplexes can favor dural ruptures and formation of diverticula, with consequent leakage. The treatment is still uncertain but considering this event a manifestation of disease activity, corticosteroids and immunosuppressants are the main therapeutic options.

\section{KEYWORDS}

Spontaneous intracranial hypotension, Systemic lupus erythematosus, Dural laceration. 